Pacific Journal of Mathematics

KATO-TAUSSKY-WIELANDT COMMUTATOR RELATIONS AND 


\title{
KATO-TAUSSKY-WIELANDT COMMUTATOR RELATIONS AND CHARACTERISTIC CURVES
}

\author{
Fergus Gaines
}

Let $A$ and $B$ be $n \times n$ matrices with elements in a field $\mathscr{F}$ and let $\Delta_{A} B=A B-B A$. Let $f_{k}(x)=x^{2 K+1}-c_{1} x^{2 K-1}+$ $c_{2} x^{2 K-3}+\cdots+(-1)^{K} c_{K} x$, where the $c_{i}$ are in $\mathscr{F}$ and $K=$ $k(k-1) / 2$. In this paper we examine the consequences of the relation $f_{k}\left(\Delta_{A}\right) B=0$, where $1 \leqq k<n$, and show how the replacement of $A$ by $x A+y B$, when $k=2$, leads to a splitting of the characteristic curve, $\operatorname{det}(x A+y B-z I)=0$, into lines and conics.

1. Introduction. We open with some notation and some definitions. Let $\mathscr{F}$ be a field and let $\mathscr{F}_{n}$ denote the $n \times n$ matrices with elements in $\mathscr{F}$. If $A$ and $B \in \mathscr{F}_{n}$, the characteristic curve of the pencil $x A+y B$ is the curve in the projective $x, y, z$-plane whose equation is $\operatorname{det}(x A+y B-z I)=0$. If $A \in \mathscr{F}_{n}$, the operator $\Delta_{A}$ is given by $\Delta_{A} X=A X-X A$, for all $X$ in $\mathscr{F}_{n}$. If $k \geqq 1$ is an integer and $K=k(k-1) / 2$ and if $c_{1}, c_{2}, \cdots, c_{K} \in \mathscr{F}$, we let $f_{k}(x)=x^{2 K+1}-$ $c_{1} x^{2 K-3}+\cdots+(-1)^{K} c_{K} x$. Next we need some ideas usually associated with the Perron-Frobenius theory of nonnegative matrices. If $X=$ $\left(x_{i j}\right) \in \mathscr{F}_{n}$, the digraph $\mathscr{G}(X)$ consists of vertices labeled $1,2,3, \cdots$, $n$ and there is an edge from $i$ to $j$, i.e. $i \rightarrow j$, if and only if $x_{i j} \neq 0$. The matrix $X \in \mathscr{F}_{n}$ is permutation-irreducible if and only if it can not be transformed by a permutation similarity to the form $\left(\begin{array}{cc}Y & Z \\ 0 & W\end{array}\right)$ where $Y$ and $W$ are square matrices.

In [4] Taussky and Wielandt proved.

THEOREM 1. If $A$ and $B \in \mathscr{F}_{n}$ and $\alpha_{1}, \alpha_{2}, \cdots, \alpha_{n}$ are the eigenvalues (in some extension field of $\mathscr{F}$ ) of $A$, then $f_{n}\left(\Delta_{A}\right) B=0$, where $c_{i}$ is the ith elementary symmetric function of the $N=n(n-1) / 2$ quantities $\left(\alpha_{r}-\alpha_{s}\right)^{2}, 1 \leqq r<s \leqq n ; i=1,2, \cdots, N$.

Since $f_{1}\left(\Delta_{A}\right) B=A B-B A$, the relation $f_{k}\left(\Delta_{A}\right) B=0$, with $1<k<$ $n$, for some $c_{1}, c_{2}, \cdots, c_{K} \in \mathscr{F}$, is a generalization of commutativity. As a generalization of matrix commutativity it is, however, quite weak, since it is still possible for $A$ and $B$ to satisfy such an identity (when $n=3$ ) and to generate $\mathscr{F}_{3}$ (see the examples in $\S 4$ ). However, it will be shown that the relation $f_{k}\left(\Delta_{A}\right) B=0$ imposes a restriction on the eigenvalues of $A$, when $A$ and $B$ generate $\mathscr{F}_{n}$. We call an expression of the form $f_{k}\left(\Delta_{A}\right) B$ a Kato-Taussky-Wielandt commutator.

We shall need one well-known result from graph theory which 
can be found, for example, in [5].

THeOREM 2. $X \in \mathscr{F}_{n}$ is permutation-irreducible if and only if $\mathscr{G}(X)$ is strongly connected.

2. The main theorem. Our principal result is

Theorem 3. Let $A$ and $B \in \mathscr{F}_{n}$ and suppose $A$ and $B$ generate $\mathscr{F}_{n}$, i.e. every matrix in $\mathscr{F}_{n}$ has the form $P(A, B)$, where $P(x, y)$ is a polynomial over $\mathscr{F}$ in the noncommuting indeterminates $x$ and $y$. If the characteristic of $\mathscr{F}$ does not divide $n$ and if, for some fixed integer $k$ with $1 \leqq k<n$, there exist $c_{1}, c_{2}, \cdots, c_{K}$ in $\mathscr{F}$ so that $f_{k}\left(\Delta_{A}\right) B=0$, then the eigenvalues of $A$ belong to the splitting field of $f_{k}(x)$ over $\mathscr{F}$.

Proof. If $k=1$ then $A B=B A$ and, since $A$ and $B$ generate $\mathscr{F}_{n}$, we get $n=1$. The theorem is then obvious.

If $A$ has only one eigenvalue $\alpha$, then $n \alpha=\operatorname{trace} A$ is in $\mathscr{F}$ and, consequently, $\alpha \in \mathscr{F}$, since the characteristic of $\mathscr{F}$ does not divide $n$.

So assume that $1<k<n$, that $A$ has at least two distinct eigenvalues and that there exist $c_{1}, c_{2}, \cdots, c_{K} \in \mathscr{F}$ so that $f_{k}\left(\Delta_{A}\right) B=$ 0 . By extending $\mathscr{F}$ to its algebraic closure $\overline{\mathscr{F}}$, we may assume (via similarity) that $A=\sum_{i=1}^{r} \oplus A_{i}$ is in Jordan canonical form, where $A_{i}$ is a direct sum of Jordan blocks, all of which have the same eigenvalue $\alpha_{i}$ and $\alpha_{i} \neq \alpha_{j}$ when $i \neq j$. We then view $B$ as a matrix over $\overline{\mathscr{F}}$ (via the similarity above) and let $B=\left(B_{i j}\right)$ be the partition of $B$ into blocks corresponding to that of $A=\sum_{i=1}^{r} \oplus A_{i}$. We shall prove

LEMMA 1. If $B_{i j} \neq 0$, then $\alpha_{i}-\alpha_{j}$ satisfies $f_{k}(x)=0$.

LeMma 2. $B=\left(B_{i j}\right)$ is permutation-irreducible as a block matrix.

If we assume these lemmas we can complete the proof of the theorem in a few lines. Let $\mathscr{G}(B)$ be the digraph of $B$ viewed as a block matrix, i.e. $i \rightarrow j$ if and only if $B_{i j} \neq 0$. Then Lemma 2 and a modification of Theorem 2 (for block matrices) imply that $\mathscr{G}(B)$ is strongly connected. Thus, if $\alpha_{i}, \alpha_{j}$ are distinct eigenvalues of $A$, there exists a sequence $i, i_{1}, i_{2}, \cdots, i_{m}, j$ so that $\alpha_{i}-\alpha_{i_{1}}, \alpha_{i_{1}}-$ $\alpha_{i_{2}}, \cdots, \alpha_{i_{m}}-\alpha_{j}$ are roots of $f_{k}(x)=0$. Let $\mathscr{L}$ be the splitting field of $f_{k}(x)$ over $\mathscr{F}$. Then

$$
\alpha_{i}-\alpha_{j}=\left(\alpha_{i}-\alpha_{i_{1}}\right)+\left(\alpha_{i_{1}}-\alpha_{i_{2}}\right)+\cdots+\left(\alpha_{i_{m}}-\alpha_{j}\right) \in \mathscr{L} .
$$


Let $n_{j}$ be the multiplicity of $\alpha_{j}$ as an eigenvalue of $A$. Then

$$
\sum_{j=1}^{r} n_{j} \alpha_{i}-\sum_{j=1}^{r} n_{j} \alpha_{j} \in \mathscr{L},
$$

i.e. $n \alpha_{i}$ - trace $A \in \mathscr{L}$. Thus $\alpha_{i} \in \mathscr{L}, i=1,2, \cdots, r$, since the characteristic of $\mathscr{L}$ does not divide $n$.

It remains to prove the lemmas to complete the proof of the theorem.

Proof of Lemma 1. We use the relation $f_{k}\left(\Delta_{A}\right) B=0$. Suppose $B_{i j} \neq 0$. Let $b_{s t}$ be the "first" nonzero element of $B_{i j}$ in the following sense: if the lower left-hand corner element of $B_{i j}$ is nonzero let it be $b_{s t}$; otherwise let $b_{s t}$ be a nonzero element of $B_{i j}$ so that $b_{u v}=0$ if $u \geqq s$ and $v \leqq t$, and $(u, v) \neq(s, t)$, where, of course, we only consider those elements $b_{u v}$ of $B_{i j}$. Thus

$$
B_{i j}=\left[\begin{array}{ccccccc} 
& & * & & & & * \\
0 & \cdot & \cdot & \cdot & 0 & b_{s t} & \\
0 & \cdot & \cdot & \cdot & 0 & 0 & \\
\cdot & & & & \cdot & \cdot & * \\
0 & \cdot & . & \cdot & 0 & 0 &
\end{array}\right] .
$$

If $b_{s t}$ is the $(s, t)$ element of $B_{i j}$ we calculate the $(s, t)$ element of the $(i, j)$ block of $f_{k}\left(A_{A}\right) B$. To simplify calculations assume that $A_{j}$ has eigenvalue zero and $A_{i}$ has eigenvalue $\alpha_{i j}=\alpha_{i}-\alpha_{j}$ (Subtract $\alpha_{j} I$ from $A$. Since we take commutators, this operation does not affect the end result of the calculations). The matrix $f_{k}\left(A_{A}\right) B$ is a linear combination of matrices of the type $\Delta_{A}^{m} B$. The $(i, j)$ block of $\Delta_{A} B$ is $A_{i} B_{i j}-B_{i j} A_{j}$. The $(i, j)$ block of $A_{A}^{m} B$ only involves $B_{i j}, A_{i}$ and $A_{j}$; it consists of a linear combination of matrices of the type $A_{i}^{c} B_{i j} A_{j}^{d}$ where $c+d=m$. The $(s, t)$ element of $A_{i}^{c} B_{i j} A_{j}^{d}$ is obtained by multiplying the $t$ th column of $B_{i j} A_{j}^{d}$ by the th row of $A_{i}^{c}$. Those elements in the $t$ th column of $B_{i j} A_{j}^{d}$ from the sth row down are all that matter here. But these elements are zero, except when $d=0$, since $A_{j}$ has zeros on and below the main diagonal. Thus the $(s, t)$ element of the $(i, j)$ block of $\Delta_{A}^{m} B$ is $\alpha_{i j}^{m} b_{s t}$. So the equation $f_{k}\left(\Delta_{A}\right) B=0$ gives $f_{k}\left(\alpha_{i j}\right)=0$, since $b_{s t} \neq 0$.

Proof of Lemma 2. Suppose there exists a block permutation matrix $Q$, partitioned conformally with $B=\left(B_{i j}\right)$, so that $Q^{-1} B Q$ has the form ( $\dagger$ ) where $m<r$. Then $A$ and $B$ are reduced by $Q$, since $Q$ simply permutes the blocks on the diagonal of $A$. Thus the algebra generated by $A$ and $B$ over $\overline{\mathscr{F}}$ is reducible. But $A$ and $B$ generate $\overline{\mathscr{F}}_{n}$. This 
contradiction proves that $B=\left(B_{i j}\right)$ is permutation-irreducible as a block matrix.

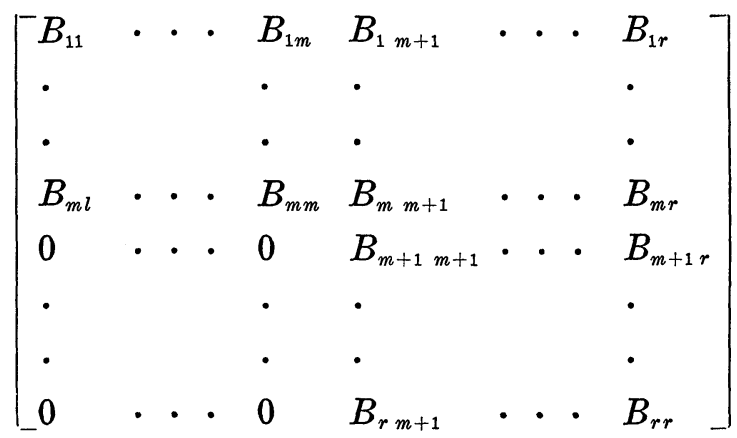

Theorem 4. Let $A$ and $B$ satisfy the conditions of Theorem 3 with $k=2$ and let $A$ have at least two distinct eigenvalues. Then there exists an ordering $\alpha_{1}, \alpha_{2}, \cdots, \alpha_{r}$ of the distinct eigenvalues of $A$ so that $\alpha_{1}-\alpha_{2}=\alpha_{2}-\alpha_{3}=\cdots=\alpha_{r-1}-\alpha_{r}$ satisfies $x^{2}-c_{1}=0$.

Proof. As in the proof of Theorem 3, let $B=\left(B_{i j}\right)$ be the block form of $B$ (over $\overline{\mathscr{F}}$ ) corresponding to the Jordan canonical form $\sum_{i=1}^{r} \oplus A_{i}$ of $A$. We have $f_{2}\left(\Delta_{A}\right) B=0$, for some $c_{1} \in \mathscr{F}$.

We claim that $B$ can not have more than two nonzero off-diagonal blocks in each row or column. For if $B_{i j}, B_{i l}$ and $B_{i m}$ are nonzero off-diagonal blocks, where $j, l$, and $m$ are distinct, then $\alpha_{i}-\alpha_{j}, \alpha_{i}-$ $\alpha_{l}$ and $\alpha_{i}-\alpha_{m}$ satisfy the equation $x^{2}-c_{1}=0$. Thus two of these $\alpha$ 's, at least, are equal, contradicting the fact that the $\alpha$ 's are distinct. If $B_{i j}$ and $B_{i l}$ (resp. $B_{j i}$ and $B_{l i}$ ) are nonzero off-diagonal blocks with $j \neq l$, and $B_{m i}\left(\right.$ resp. $\left.B_{i m}\right)$ is also a nonzero off-diagonal block, a similar argument proves $m=j$ or $m=l$.

Let $\mathscr{G}(B)$ be the digraph of $B$ viewed as a block matrix. We write $i \sim j$ if $i \rightarrow j$ or $j \rightarrow i$. So if $i \sim j$ and $i \sim l$, where $i, j$ and $l$ are distinct, then $i \sim m$ implies either $j$ or $l$ is $m$. We claim that, by relabeling the vertices of $\mathscr{G}(B)$, we get the subgraph

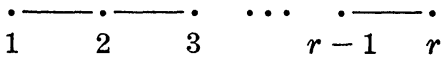

where $i \sim i+1$, for $i=1,2, \cdots, r-1$. For let

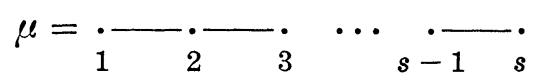

be a maximal "path" in $\mathscr{G}(B)$ (on relabeling vertices), where $i \sim$ $i+1$ for $i=1,2, \cdots, s-1$, and suppose $s \neq r$. If $j$ is a vertex not in $\mu$ then neither $j \sim 1$ nor $j \sim s$ can hold, since $\mu$ is maximal. Since $\mathscr{G}(B)$ is strongly connected, there exists an internal vertex $i \in \mu$ 
and a vertex $j \notin \mu$ so that $i \sim j$. But $i \sim i+1$ and $i \sim i-1$ and, since neither or these is $j$, we get a contradiction. Thus $\mathscr{G}(B)$ contains the required subgraph. By Lemma 1 , this means the distinct eigenvalues $\alpha_{i}$ of $A$ can be relabeled so that $\alpha_{1}-\alpha_{2}, \alpha_{2}-\alpha_{3}, \cdots, \alpha_{r-1}-\alpha_{r}$ satisfy the equation $x^{2}-c_{1}=0$. Since the $\alpha$ 's are distinct, we get

$$
\alpha_{i}-\alpha_{i+1}=\alpha_{i+1}-\alpha_{i+2}, \quad 1=1,2, \cdots \text {. }
$$

This completes the proof of the theorem.

3. Generalized $L$-property. Let $A$ and $B$ be $n \times n$ matrices with elements in $\mathscr{F}$ and suppose the eigenvalues of $A$ and $B$ are also in $\mathscr{F}$. If there exist fixed orderings $\alpha_{1}, \alpha_{2}, \cdots, \alpha_{n}$ and $\beta_{1}, \beta_{2}$, $\cdots, \beta_{n}$ of the eigenvalues of $A$ and $B$, respectively, so that the eigenvalues of $x A+y B$ are $x \alpha_{i}+y \beta_{i}$, for all $x$ and $y$ in $\mathscr{F}$, where $i=1,2, \cdots, n$, then $A$ and $B$ have property $L$. Property $L$ has been discussed by Motzkin and Taussky [3]; it is clearly equivalent to the assertion that the characteristic curve of the pencil $x A+y B$ is the union of $n$ lines (if $\mathscr{F}$ is big enough). In this section we discuss a condition which forces the characteristic curve to decompose into lines and conices.

Let $\mathscr{F}[x, y]$ be the integral domain of polynomials over $\mathscr{F}$ in the (commuting) indeterminates $x$ and $y$. Let $\mathscr{F}(x, y)$ be its quotient field.

LEMMA 3. Let $p(x, y, z)$ be a homogeneous polynomial in $x, y$ and $z$, with coefficients in $\mathscr{F}$. Suppose

$$
p(x, y, z)=\prod_{i=1}^{r} p_{i}^{k} i,
$$

where each $p_{i}$ is an irreducible polynomial in $z$ over $\mathscr{F}(x, y)$. Then each $p_{i}$ is a homogeneous polynomial in $x, y$, and $z$, with ooefficients in $\mathscr{F}$.

Proof. $\mathscr{F}[x, y]$ is a unique factorization domain (UFD). Since a UFD is integrally closed ([2], p. 84), the coefficients of the powers of $z$ in $p_{i}$ must be polynomials in $x$ and $y$.

Suppose $p_{i}$ is not homogeneous in $x, y$ and $z$. Let $M(q)$ (resp. $m(q)$ ) be the maximum (resp. minimum) degree of the monomials in a polynomial $q$. Then $M\left(p_{i}\right)>m\left(p_{i}\right)$ and $M$ (resp. $m$ ) has the property that $M\left(q_{1} q_{2}\right)=M\left(q_{1}\right)+M\left(q_{2}\right)\left(m\left(q_{1} q_{2}\right)=m\left(q_{1}\right)+m\left(q_{2}\right)\right)$ for polynomials $q_{1}$ and $q_{2}$. Hence $M(p)>m(p)$, which is false. The Lemma is proved.

We now apply the results of $\S 2$ to $x A+y B$ and $B$.

THEOREM 5. Let $A$ and $B \in \mathscr{F}_{n}$, where $\mathscr{F}$ is an infinite field 
whose characteristic does not divide $n$. If $A$ and $B$ generate $\mathscr{F}_{n}$ and if, for each $x$ and $y$ in $\mathscr{F}$, there exists $c_{1}$ in $\mathscr{F}$ so that

$$
f_{2}\left(\Delta_{x A+y B}\right) B=0,
$$

then the characteristic polynomial $p(x, y, z)$ of $x A+y B$ splits into linear and quadratic homogeneous factors with coefficients in $\mathscr{F}$.

Proof. Without loss, we may assume that $n \geqq 3$. Let $X=x A+y B$. If $\Delta_{X} B=0$, for $x \neq 0$, then $A B=B A$, which implies $n=1$. So $\Delta_{X} B \neq 0$ (for $x \neq 0$ ) and the relation $f_{2}\left(\Delta_{X}\right) B=0$ imply that $c_{1}$ is a rational function of $x$ and $y$. Since $\mathscr{F}$ is infinite, we may replace $x$ and $y$ by two algebraically independent indeterminates and the relation $f_{2}\left(\Delta_{X}\right) B=0$ still holds. $X$ and $B$ clearly generate $\mathscr{F}(x, y)_{n}$, and thus we may apply Theorem 3. Since $f_{2}(w)=w^{3}-c_{1} w$, each eigenvalue of $X=x A+y B$ satisfies an equation of degree at most 2 over $\mathscr{F}(x, y)$. Lemma 3 is now used to complete the proof.

CoROLlaRY. Let $\mathscr{F}$ be an algebraically closed field of characteristic zero or greater than $n$. If $A$ and $B \in \mathscr{F}_{n}$ and if, for each $x$ and $y \in \mathscr{F}$, there exists $c_{1} \in \mathscr{F}$ so that

$$
f_{2}\left(\Delta_{x A+y B}\right) \in \mathcal{J},
$$

where $\mathcal{J}$ is the radical of the algebra generated by $A$ and $B$ over $\mathscr{F}$, then the characteristic polynomial $p(x, y, z)$ of $x A+y B$ splits into linear and quadratic homogeneous factors in $x, y$ and $z$ with coefficients in $\mathscr{F}$.

Proof. If $A_{1}$ and $B_{1}$ are the representatives of $A$ and $B$ respectively, in an irreducible representation of the algebra generated by $A$ and $B$ over $\mathscr{F}$, then $f_{2}\left(\Delta_{X_{1}}\right) B_{1}=0$, where $X_{1}=x A_{1}+y B_{1}$. Also $A_{1}$ and $B_{1}$ generate a complete matrix algebra, since $\mathscr{F}$ is algebraically closed. $A$ and $B$ may be transformed by a similarity into block upper triangular form, where the corresponding diagonal blocks generate irreducible matrix algebras. The conclusion follows.

REMARK. Nothing we have said so far forces the characteristic curve of $x A+y B$ to contain a line. If $\mathscr{F}$ has characteristic zero or greater than $n$ and $f_{2}\left(\Delta_{x_{A+y B}}\right) B=0$ for some $c_{1} \in \mathscr{F}(x, y)$, where $x$ and $y$ are algebraically independent over $\mathscr{F}$, and if $x A+y B$ has an odd number of distinct eigenvalues, then at least one of the eigenvalues has the form $x \alpha+y \beta$, where $\alpha, \beta \in \mathscr{F}$. For let $z_{1}, z_{2}$, $\cdots, z_{r}$ be the distinct eigenvalues of $x A+y B$, with $r$ odd. If $r=$ 1 the result is trivial. Let $r \geqq 3$; then, by Theorem 4 , we may assume 
the eigenvalues are ordered so that $z_{1}-z_{2}=z_{2}-z_{3}=\cdots z_{r-1}-z_{r}$ satisfies $w^{2}-c_{1}=0$. By the condition on the characteristic of $\mathscr{F}$, the irreducible factors of $p(x, y, z)$ - the characteristic polynomial of $x A+y B-$ are separable. Hence $\sum_{i=1}^{r} z_{i} \in \mathscr{F}(x, y)$. Now

$$
(1 / r) \sum_{i=1}^{r} z_{i}=z_{r}+((r-1) / 2) c_{1}^{1 / 2},
$$

since $z_{i}-z_{i+1}=c_{1}^{1 / 2}, i=1,2, \cdots, r-1$. Since $r$ is odd, $(r-1) / 2=s$ is an integer and $z_{r-s}=z_{r}+((r-1) / 2) c_{1}^{1 / 2}$ is in $\mathscr{F}(x, y)$. By Lemma $3, z_{r-s}=x \alpha+y \beta$, where $\alpha$ and $\beta \in \mathscr{F}$.

\section{Examples.}

EXAMPLE 1. This example illustrates the main results of the paper. Let $\mathscr{F}$ be a field of characteristic not 2 or 3 . Let

$$
A=\left[\begin{array}{lll}
1 & 1 & 0 \\
1 & 1 & 1 \\
0 & 1 & 1
\end{array}\right] \text { and } B=\left[\begin{array}{lll}
1 & 0 & 0 \\
0 & 2 & 0 \\
0 & 0 & 3
\end{array}\right]
$$

Then $A$ and $B$ generate $\mathscr{F}_{3}$. If $X=x A+y B$, where $x$ and $y$ are algebraically independent over $\mathscr{F}$, then $X$ and $B$ generate $\mathscr{F}(x, y)_{3}$ and, if $c_{1}=\sqrt{2 x^{2}+y^{2}}$, then $f_{2}\left(\Delta_{X}\right) B=0$. The characteristic polynomial of $x A+y B$ is

$$
(x+2 y-z)\left(z^{2}-(2 x+4 y) z-x^{2}+4 x y+3 y^{2}\right)
$$

(cf. Theorem 5). The eigenvalues of $x A+y B$ are

$$
\begin{gathered}
z_{2}=x+2 y \\
z_{1}=x+2 y+\sqrt{2 x^{2}+y^{2}}, \quad z_{3}=x+2 y-\sqrt{2 x^{2}+y^{2}} .
\end{gathered}
$$

Clearly

$$
z_{1}-z_{2}=z_{2}-z_{3}=\sqrt{2 x^{2}+y^{2}}
$$

(cf. Theorem 4). We see that

$$
z_{1}, z_{2}, z_{3} \in \mathscr{F}\left(x, y, \sqrt{\left.2 x^{2}+y^{2}\right)}\right.
$$

(cf. Theorem 3).

EXAMPLE 2. The example we give here is a counterexample to Theorem 3 and Theorem 5, when the condition on the characteristic of $\mathscr{F}$ is not satisfied. Let $\mathscr{F}$ have characteristic 3 and let 


$$
A=\left[\begin{array}{rrr}
0 & -1 & -1 \\
1 & 0 & 1 \\
1 & -1 & 0
\end{array}\right] \text { and } B=\left[\begin{array}{lll}
0 & 1 & 0 \\
0 & 0 & 1 \\
0 & 0 & 0
\end{array}\right]
$$

Then $A$ and $B$ generate $\mathscr{F}_{3}$ and, if $X=x A+y B$ and $c_{1}=0$, then $f_{2}\left(\Delta_{X}\right) B=0$. Now $f_{2}(w)=w^{3}$ and the characteristic polynomial of $x A+y B$ is $x y^{2}-z^{3}=\left(\left(x y^{2}\right)^{1 / 3}-z\right)^{3}$. Theorems 3 and 5 clearly fail here.

Acknowledgments. The results in this paper are contained in the last chapter of the author's Ph. D. thesis (Caltech, 1966), which was directed by Olga Taussky Todd. Sincere thanks are offered to her for much helpful advice; thanks are also due to E. C. Dade. A special word of thanks to Ed Bender for a helpful conversation on the material of this paper.

\section{REFERENCES}

1. F. Gaines, Kato-Taussky-Wielandt commutator relations, Linear Algebra and its Applications, I (1968), 127-138.

2. N. Jacobson, Lectures in Abstract Algebra, I, Van Nostrand, Princeton, 1951.

3. T. S. Motzkin and O. Taussky, Pairs of matrices with property L, I, Trans. Amer. Math. Soc., 73 (1952), 108-114; II, ibid., 80 (1955), 387-401.

4. $\mathrm{O}$. Taussky and $\mathrm{H}$. Wielandt, Linear relations between higher additive commutators, Proc. Amer. Math. Soc., 13 (1962), 732-735.

5. R. S. Varga, Matrix Iterative Analysis, Prentice-Hall, Inc., Englewood Cliffs, New Jersey, 1962.

Received March 15, 1975. Research supported in part by a grant from the National Science Foundation.

University College Dublin 4, Ireland 


\section{PACIFIC JOURNAL OF MATHEMATICS}

\section{EDITORS}

RICHARD ARENS (Managing Editor) University of California

Los Angeles, California 90024

\section{R. A. BEAUMONT}

University of Washington Seattle, Washington 98105
J. DugundjI Department of Mathematics University of Southern Californı Los Angeles, California 90007

D. Gilbarg AND J. Milgram Stanford University Stanford, California 94305

\section{ASSOCIATE EDITORS}

E. F. BECKENBACH

B. H. NeUMANN

F. WOLF

K. YosHidA

\section{SUPPORTING INSTITUTIONS}

UNIVERSITY OF BRITISH COLUMBIA CALIFORNIA INSTITUTE OF TECHNOLOGY UNIVERSITY OF CALIFORNIA MONTANA STATE UNIVERSITY UNIVERSITY OF NEVADA NEW MEXICO STATE UNIVERSITY OREGON STATE UNIVERSITY UNIVERSITY OF OREGON OSAKA UNIVERSITY

\author{
UNIVERSITY OF SOUTHERN CALIFORNIA \\ STANFORD UNIVERSITY \\ UNIVERSITY OF TOKYO \\ UNIVERSITY OF UTAH \\ WASHINGTON STATE UNIVERSITY \\ UNIVERSITY OF WASHINGTON \\ AMERICAN MATHEMATICAL SOCIETY \\ NAVAL WEAPONS CENTER
}




\section{Pacific Journal of Mathematics}

\section{Vol. 61, No. $1 \quad$ November, 1975}

Jiří Adámek, V. Koubek and Věra Trnková, Sums of Boolean spaces represent every

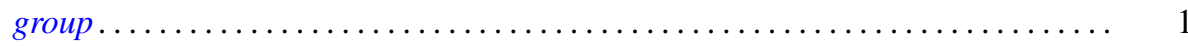

Richard Neal Ball, Full convex l-subgroups and the existence of $a^{*}$-closures of

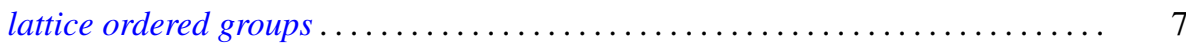

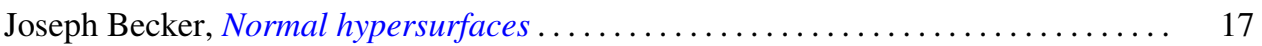

Gerald A. Beer, Starshaped sets and the Hausdorff metric . . . . . . . . . . . . . 21

Dennis Dale Berkey and Alan Cecil Lazer, Linear differential systems with

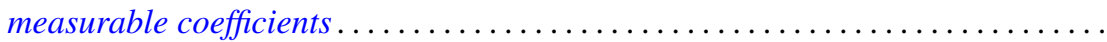

Harald Boehme, Glättungen von Abbildungen 3-dimensionaler

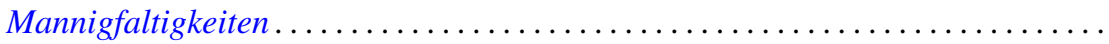

Stephen LaVern Campbell, Linear operators for which $T^{*} T$ and $T+T^{*}$

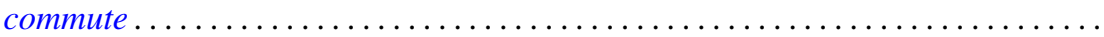

H. P. Dikshit and Arun Kumar, Absolute summability of Fourier series with

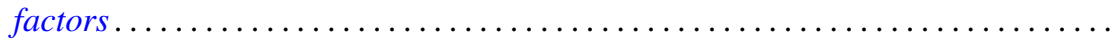

Andrew George Earnest and John Sollion Hsia, Spinor norms of local integral

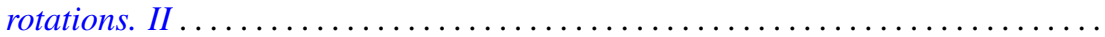

Erik Maurice Ellentuck, Semigroups, Horn sentences and isolic structures .........

Ingrid Fotino, Generalized convolution ring of arithmetic functions . . . . . . . . . . .

Michael Randy Gabel, Lower bounds on the stable range of polynomial rings .......

Fergus John Gaines, Kato-Taussky-Wielandt commutator relations and

characteristic curves

Theodore William Gamelin, The polynomial hulls of certain subsets of $C^{2}$

R. J. Gazik and Darrell Conley Kent, Coarse uniform convergence spaces. . .

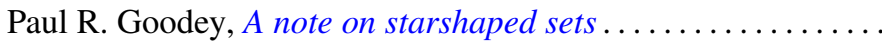

Eloise A. Hamann, On power-invariance

M. Jayachandran and M. Rajagopalan, Scattered compactification for $N \cup\{P\}$. . .

V. Karunakaran, Certain classes of regular univalent functions .

John Cronan Kieffer, A ratio limit theorem for a strongly subadditive set function in a locally compact amenable group .................

Siu Kwong Lo and Harald G. Niederreiter, Banach-Buck measure, density, and uniform distribution in rings of algebraic integers ........

Harold W. Martin, Contractibility of topological spaces onto metric spaces ....

Harold W. Martin, Local connectedness in developable spaces .

A. Meir and John W. Moon, Relations between packing and covering numbers of a tree.

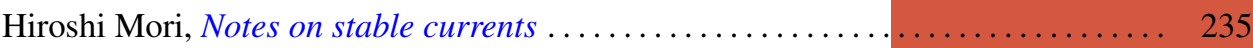

Donald J. Newman and I. J. Schoenberg, Splines and the logarithmic function . . . . 241

M. Ann Piech, Locality of the number of particles operator....

Fred Richman, The constructive theory of $K T$-modules .......

Gerard Sierksma, Carathéodory and Helly-numbers of

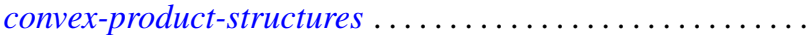

Raymond Earl Smithson, Subcontinuity for multifunctions .... . . 